\title{
Safe and Near-Optimal Policy Learning for Model Predictive Control using Primal-Dual Neural Networks
}

\author{
Xiaojing Zhang, Monimoy Bujarbaruah, and Francesco Borrelli
}

\begin{abstract}
In this paper, we propose a novel framework for approximating the explicit MPC law for linear parametervarying systems using supervised learning. In contrast to most existing approaches, we not only learn the control policy, but also a "certificate policy", that allows us to estimate the sub-optimality of the learned control policy online, during execution-time. We learn both these policies from data using supervised learning techniques, and also provide a randomized method that allows us to guarantee the quality of each learned policy, measured in terms of feasibility and optimality. This in turn allows us to bound the probability of the learned control policy of being infeasible or suboptimal, where the check is performed by the certificate policy. Since our algorithm does not require the solution of an optimization problem during runtime, it can be deployed even on resource-constrained systems. We illustrate the efficacy of the proposed framework on a vehicle dynamics control problem where we demonstrate a speedup of up to two orders of magnitude compared to online optimization with minimal performance degradation.
\end{abstract}

\section{INTRODUCTION}

Model Predictive Control (MPC) is an advanced control strategy that is able to optimize a plant's behavior while respecting system constraints. Originating in process control, MPC has found application in fields such as building climate control [1]-[3], quadcopter control [4], self-driving vehicles [5]-[10] and robotics in general [11]. However, implementing MPC on fast dynamical systems with limited computation capacity is generally challenging since MPC requires the solution of an optimization problem at each sampling step. This is especially true for mass-produced systems such as drones and automotive vehicles.

Over the past decades, significant research effort has been devoted to enabling MPC to systems with limited computation power by developing numerically efficient solvers that exploit the structure of the MPC optimization problem [12], [13]. Another approach to reduce computation load of MPC is to pre-compute the optimal control law offline, store it into the system, and evaluate it during run-time. This approach, known as Explicit $M P C$, is generally well-understood for linear time-invariant system where the optimal control law has been shown to be piecewise affine over polyhedral regions [14]. The main drawbacks of Explicit MPC is that synthesis of the optimal control law can be computationally demanding even for medium-sized problems, and storing and evaluating the look up tables can be prohibitive for embedded platforms [15]. To address this issue, significant effort has been devoted to computing suboptimal explicit MPC polices that are defined over fewer polyhedral regions and hence

The authors are with the MPC Lab, UC Berkeley, USA; E-mails: \{xiaojing.zhang, monimoyb, fborrelli\}@berkeley.edu. can be evaluated more efficiently [16]-[20]. Another way of approximating explicit MPC control laws is by means of function approximation such as supervised learning [21] or reinforcement learning [22]. In supervised learning, for example, the goal is to find a function, out of a given function class, that best explains some given training data. One main advantage of using such function approximation is that, while training can be computationally demanding, evaluating the control law can often be carried out very efficiently [23], [24]. Although techniques such as supervised learning can in principle apply to nonlinear systems, most research has focused on linear time-invariant systems [22]-[25], since guaranteeing safety and performance of the approximated control law is generally hard for nonlinear systems [26].

In this paper, we propose a novel policy approximation scheme for learning the explicit MPC control law using a primal policy and a dual policy. The policies are trained and verified offline using randomly generated training and verification data, respectively. Online, in real-time, the dual policy is then used to estimate the performance of the control action given by the primal policy. This is in contrast to most existing methods that incorporate safety constraints during the policy learning phase, which may result in suboptimal controllers. Specifically, our contributions can be summarized as follows:

- For the offline phase, we propose a supervised learning scheme to train the primal and dual policies, and introduce a randomized verification methodology to estimate the quality (i.e., feasibility and suboptimality) of the trained policies. Given an admissible probability of quality violation, explicit sample sizes are provided for the verification step.

- We show how, during the online phase, we can use the dual policy to track the quality (i.e., feasibility and suboptimality) of the approximated MPC law i.e. the primal policy, using ideas from duality theory of convex optimization. If the check fails, then a backup controller is used.

- We demonstrate the efficacy of the proposed primaldual policy learning framework for an integrated chassis control problem in vehicle dynamics. In particular, we demonstrate computation speedups of up to $100 \mathrm{x}$, when compared to state-of-the-art numerical solvers, potentially enabling the implementation of MPC on mass-produced embedded systems.

In contrast to most existing methods, our methodology also applies to linear parameter-varying systems. We also stress that the proposed framework is applicable to any function 
approximation scheme. In that regard, our methodology is able to check safety and performance of control laws that are encoded through deep neural networks (DNN).

\section{PROBLEM DESCRIPTION}

\section{A. Dynamics, constraints, and control objective}

We consider linear parameter-varying (LPV) systems of the form

$$
x_{k+1}=A\left(q_{k}\right) x_{k}+B\left(q_{k}\right) u_{k},
$$

where $x_{k} \in \mathbb{R}^{n_{x}}$ is the state at time $k, u_{k} \in \mathbb{R}^{n_{u}}$ is the input at time $k$, and $A\left(q_{k}\right)$ and $B\left(q_{k}\right)$ are known matrices of appropriate dimensions, that depend on a time-varying parameter $q_{k}$. Throughout this paper, we assume that the parameter $q_{k}$ is known. The system is subject to linear input and state constraints of the form

$$
\mathbb{U}:=\left\{u: H_{u} u_{k} \leq h_{u}\right\}, \quad \mathbb{X}:=\left\{x: H_{x} x_{k} \leq h_{x}\right\},
$$

for given $H_{u}, h_{u}, H_{x}$ and $h_{x}$. At each time step $k$, the control objective is to minimize, over a finite horizon $T$, a quadratic cost of the form

$$
x_{T}^{\top} Q_{f} x_{T}+\sum_{k=0}^{T-1} x_{k}^{\top} Q x_{k}+u_{k}^{\top} R u_{k},
$$

where the matrices $Q$ and $Q_{f}$ are assumed to be positive semi-definite and $R$ is chosen to be positive definite.

\section{B. Model Predictive Control}

At each time step $t$, Model Predictive Control (MPC) measures the state $x_{t}$ and solves the following finite-horizon optimal control problem

$$
\begin{array}{cl}
\min _{U_{t}, X_{t}} & x_{N \mid t}^{\top} Q_{f} x_{T \mid t}+\sum_{k=0}^{T-1} x_{k \mid t}^{\top} Q x_{k \mid t}+u_{k \mid t}^{\top} R u_{k \mid t} \\
\text { s.t. } & x_{k+1 \mid t}=A\left(q_{k \mid t}\right) x_{k \mid t}+B\left(q_{k \mid t}\right) u_{k \mid t}, \\
& \left(x_{k \mid t}, u_{k \mid t}\right) \in \mathbb{X} \times \mathbb{U}, x_{T \mid t} \in \mathbb{X}_{f}, \\
& x_{0 \mid t}=x_{t}, \quad k=0, \ldots, T-1,
\end{array}
$$

where $x_{k \mid t}$ is the state at time $t+k$ obtained by applying the predicted inputs $u_{0 \mid t}, \ldots, u_{k-1 \mid t}$ to system (1). Furthermore, $U_{t}:=\left[u_{0 \mid t}, \ldots, u_{T-1 \mid t}\right]$ and $X_{t}:=\left[x_{0 \mid t}, \ldots, x_{T \mid t}\right]$ are the collection of all predicted inputs and states, respectively. The set $\mathbb{X}_{f} \subset \mathbb{R}^{n_{x}}$, which we assume is a compact polytope, is a so-called terminal set, and ensures recursive feasibility of the MPC controller, see e.g. [14] for details. If $U_{t}^{*}$ is a minimizer of (4), then MPC applies the first input $u_{t}=u_{0 \mid t}^{*}$. This process is repeated at the next time step, resulting in a receding horizon control scheme.

By eliminating the states $X_{t}$ from (4), we can express (4) compactly as

$$
\begin{aligned}
J^{*}\left(P_{t}\right):=\min _{U} & \frac{1}{2} U^{\top} Q\left(P_{t}\right) U+c\left(P_{t}\right)^{\top} U \\
\text { s.t. } & H\left(P_{t}\right) U \leq h\left(P_{t}\right),
\end{aligned}
$$

where $P_{t}:=\left[x_{t}, q_{0 \mid t}, \ldots, q_{T-1 \mid t}\right]$ is the collection of all parameters $\left\{q_{k \mid t}\right\}_{k}$ and the initial state, and $Q\left(P_{t}\right)$, $c\left(P_{t}\right), H\left(P_{t}\right), h\left(P_{t}\right)$ are appropriately defined matrices, see e.g., [27] for their construction. It is assumed that, at each time step $t$, the parameters $P_{t}$ are known. In practice, they may come from an external estimator. We point out that (5) is a multi-parametric quadratic program, whose optimizer $U^{*}(\cdot)$ and optimal value $J^{*}(\cdot)$ depend on $P_{t}[14]$. To streamline the upcoming presentation, we assume in this paper that the parameters $P_{t}$ take values in a compact set $\mathcal{P}$, and that (5) is feasible and finite for all $P_{t} \in \mathcal{P}$.

Solving the optimization problem (5) at each sampling time can be computationally challenging for fast systems on resource constrained platforms. To address this issue, we propose the use of function approximation to offline learn an approximate policy $\tilde{U}_{\theta}(\cdot) \approx U^{*}(\cdot)$ ("primal policy"), as well as an run-time optimality certificate via a so-called "dual policy".

\section{TECHNICAL BACKGROUND}

\section{A. Supervised Learning}

The goal in classical function approximation is to approximate a function $f \in \mathcal{F}$, defined on some function space $\mathcal{F}$, by another function $\tilde{f} \in \tilde{\mathcal{F}} \subset \mathcal{F}$ such that $\|f-\tilde{f}\|_{\mathcal{F}}$ is minimized. Since this minimization problem is performed over the infinite dimensional space of functions $\tilde{\mathcal{F}}$, it is generally intractable. A common approach is to restrict oneself to function spaces $\tilde{\mathcal{F}}=\tilde{\mathcal{F}}_{\theta}$ that are defined by a finite number of parameters $\theta$, and to approximate the norm $\|\cdot\|_{\mathcal{F}}$ by the empirical error. This is achieved by drawing $M$ samples $\left\{z^{(i)}, f\left(z^{(i)}\right)\right\}_{i=1}^{M}$, upon which the finite-dimensional problem of learning $f(\cdot)$ is given by

$$
\theta^{*}:=\arg \min _{\theta} \sum_{i=1}^{M} \mathcal{L}\left(f\left(z^{(i)}\right)-\tilde{f}_{\theta}\left(z^{(i)}\right)\right),
$$

where $\theta^{*}$ denotes the optimal parameter and $\mathcal{L}(\cdot)$ a loss function, such as the euclidean norm. The choice of the loss function and the function space $\tilde{\mathcal{F}}_{\theta}$ is often problemdependent. Typical function classes include Deep Neural Networks and weighted sums of basis functions, see [28], [29] for examples.

\section{B. Duality Theory}

Duality is used in optimization to certify optimality of a given (candidate) solution. Specifically, to every convex optimization problem $p^{*}:=\min _{x}\{f(x): h(x) \leq 0\}$, we can associate its dual problem $d^{*}:=\max _{\lambda}\{g(\lambda): \lambda \geq 0\}$, where $g(\lambda):=\min _{x}\left\{f(x)-\lambda^{\top} h(x)\right\}$. Under appropriate technical assumptions 1 , it can be shown that $p^{*}=d^{*}$ ("strong duality"). In this paper, we will make use of the weak duality property that, for every primal feasible point $x$ and every dual feasible $\lambda$, it holds

$$
g(\lambda) \leq f(x) .
$$

Notice that (7) can be used to bound the suboptimality of a candidate solution $\bar{x}$, since $f(\bar{x})-p^{*} \leq f(\bar{x})-g(\lambda)$, for any $\lambda \geq 0$.

\footnotetext{
${ }^{1}$ These include feasibility, finite optimal value, and constraint qualifications, see [30. Chapter 5] for details.
} 
Dual of (5): It is well-known that the dual of a convex quadratic optimization problem is again a convex quadratic optimization problem [30]. Specifically, the dual of [5], is given by

$$
\begin{aligned}
D^{*}\left(P_{t}\right):=\min _{\lambda_{t}} & \frac{1}{2} \lambda_{t}^{\top} \tilde{Q}\left(P_{t}\right) \lambda_{t}+\tilde{c}\left(P_{t}\right)^{\top} \lambda_{t}+\tilde{g}\left(P_{t}\right) \\
\text { s.t. } & \lambda_{t} \geq 0,
\end{aligned}
$$

where $\tilde{Q}(\cdot), \tilde{c}(\cdot)$ and $\tilde{g}(\cdot)$ depend on $P_{t}$. Notice that, similar to (5), the optimizer of (8) depends on the parameters $P_{t}$, i.e, $\lambda_{t}^{*}=\lambda_{t}^{*}\left(P_{t}\right)$. Furthermore, since (5) is convex, it follows from strong duality that $J^{*}\left(P_{t}\right)=D^{*}\left(P_{t}\right)$.

\section{Primal-Dual Policy Learning}

In this section, we present our primal-dual policy learning framework, where we learn both a primal policy $\tilde{U}_{\theta_{p}}(\cdot) \approx$ $U^{*}(\cdot)$ and a dual policy $\tilde{\lambda}_{\theta_{d}}(\cdot) \approx \lambda^{*}(\cdot)$. We show how these approximated functions can be used to efficiently ensure feasibility and near-optimality of the control law during runtime of the controller.

\section{A. Primal and Dual Learning Problems}

We use supervised learning to approximate the primal policy $U^{*}(\cdot)$ and dual policy $\lambda^{*}(\cdot)$. To this end, we generate $M$ samples $\left\{P^{(i)}, U^{*}\left(P^{(i)}\right), \lambda^{*}\left(P^{(i)}\right)\right\}_{i=1}^{M}$, where $P^{(i)} \in \mathcal{P}$ are extracted according to some user-chosen distribution $\mathbb{P}$, and $U^{*}\left(P^{(i)}\right)$ and $\lambda^{*}\left(P^{(i)}\right)$ are obtained by solving (5) and (8), respectively. The choice of the distribution $\mathbb{P}$ in general will depend on the task. One could, for example, bias the distribution around a nominal operating point. If no such operating point is known, then the uniform distribution over $\mathcal{P}$ can be chosen.

Given the samples, the primal learning problem is

$$
\theta_{p}^{*}:=\arg \min _{\theta_{p}} \sum_{i=1}^{M} \mathcal{L}\left(\tilde{U}_{\theta_{p}}\left(P^{(i)}\right)-U^{*}\left(P^{(i)}\right)\right),
$$

while the dual learning problem is given by

$$
\theta_{d}^{*}:=\arg \min _{\theta_{d}} \sum_{i=1}^{M} \mathcal{L}\left(\tilde{\lambda}_{\theta_{d}}\left(P^{(i)}\right)-\lambda^{*}\left(P^{(i)}\right)\right) .
$$

We refer to $\tilde{U}_{\theta_{p}^{*}}(\cdot)$ and $\tilde{\lambda}_{\theta_{d}^{*}}(\cdot)$ as the approximated primal policy and approximated dual policy, respectively. Depending on the choice of the learning algorithm, problems 9a and $9 \mathrm{~b}$ can be computationally demanding to solve. Hence, those optimization problems are generally carried out offline.

In general once $9 \mathrm{a}$ and $9 \mathrm{~b}$ have been solved offline, it is difficult to validate feasibility and optimality of the approximated policies $\tilde{U}_{\theta_{p}^{*}}(\cdot)$ and $\tilde{\lambda}_{\theta_{p}^{*}}(\cdot)$. In the following, we describe a sampling based probabilistic verification scheme (offline, before deployment, Section IV-B], and a "hard" deterministic verification scheme (online, during run-time, Section IV-C).

\section{B. Probabilistic Safety and Performance Guarantees}

In this section, we provide a methodology to verify the feasibility and optimality of the approximated policies offline after 9a and (9b) are solved. Specifically, given a desired maximum suboptimality level, we would like to verify that the approximated primal policy satisfies this suboptimality level with high probability. Formally, we define the primal and dual objective functions

$$
\begin{aligned}
p(P ; U) & :=\frac{1}{2} U^{\top} Q(P) U+c(P)^{\top} U \\
d(P ; \lambda) & :=\frac{1}{2} \lambda^{\top} \tilde{Q}(P) \lambda+\tilde{c}(P)^{\top} \lambda+\tilde{g}(P) .
\end{aligned}
$$

Our goal is to ensure that the approximated policies are feasible and near-optimal with high probability, i.e.,

$$
\begin{array}{ll}
\mathbb{P}[ & H(P) \tilde{U}_{\theta_{p}^{*}}(P) \leq h(P), \\
& \left.p\left(P ; \tilde{U}_{\theta_{p}^{*}}(P)\right) \leq J^{*}(P)+\gamma_{p}\right] \geq 1-\epsilon_{p}, \\
\mathbb{P}[ & \tilde{\lambda}_{\theta_{d}^{*}}(P) \geq 0, \\
& \left.d\left(P ; \tilde{\lambda}_{\theta_{d}^{*}}(P)\right) \geq J^{*}(P)-\gamma_{d}\right] \geq 1-\epsilon_{d},
\end{array}
$$

where $\gamma_{p}\left(\gamma_{d}\right)$ are user-defined desired primal (dual) suboptimality levels, $\epsilon_{p}\left(\epsilon_{d}\right)$ are the user-defined maximum admissible primal (dual) violation probabilities, and $J^{*}(P)=$ $p\left(P ; U^{*}(P)\right)=d\left(P ; \lambda^{*}(P)\right)=D^{*}(P)$ denotes the optimal value.

In general, evaluating (11a)-(11b) is difficult since a multidimensional probability integral needs to be evaluated. In the following, we propose a sampling based method for verifying the above conditions. To this end, we extract $N_{p}$ primal and $N_{d}$ dual (verification) samples $\left\{P^{(i)}, J^{*}\left(P^{(i)}\right)\right\}_{i}$.

Proposition 1: Let $0<\beta_{p} \ll 1$ and $0<\beta_{d} \ll 1$ be desired primal and dual confidence levels, and $N_{p} \geq$ $\frac{\ln \left(1 / \beta_{p}\right)}{\ln \left(1 /\left(1-\epsilon_{p}\right)\right)}, N_{d} \geq \frac{\ln \left(1 / \beta_{d}\right)}{\ln \left(1 /\left(1-\epsilon_{d}\right)\right)}$. If the following "primal conditions" hold

$$
\left.\begin{array}{l}
H\left(P^{(i)}\right) \tilde{U}_{\theta_{p}^{*}}\left(P^{(i)}\right) \leq h\left(P^{(i)}\right), \\
p\left(P^{(i)} ; \tilde{U}_{\theta_{p}^{*}}\left(P^{(i)}\right)\right) \leq J^{*}\left(P^{(i)}\right)+\gamma_{p}
\end{array}\right\} i=1, \ldots, N_{p}
$$

then (11a) is satisfied with confidence at least $1-\beta_{p}$. Similarly, if the following "dual conditions" hold

$$
\left.\begin{array}{l}
\tilde{\lambda}_{\theta_{d}^{*}}\left(P^{(i)}\right) \geq 0, \\
d\left(P^{(i)} ; \tilde{\lambda}_{\theta_{d}^{*}}\left(P^{(i)}\right)\right) \geq J^{*}\left(P^{(i)}\right)-\gamma_{d}
\end{array}\right\} i=1, \ldots, N_{d},
$$

then $11 \mathrm{~b}$ is satisfied with confidence at least $1-\beta_{d}$.

Proof: The proof is based on [31, Theorem 3.1]. Details are provided in the Appendix.

Given $\epsilon_{p / d}, \gamma_{p / d}, \beta_{p / d}>0$, Proposition 1 lower bounds the sample sizes $N_{p}$ and $N_{d}$ that need to be used for verification of the trained policies. The so-called confidence levels $\beta_{p / d}$ are typically chosen very small $\left(10^{-6} \sim 10^{-8}\right)$, and only have a small impact on the sample sizes due to the logdependence. Intuitively, they bound the probability of the verification schemes failing, see [32] for details. In practice, Proposition 1 can be used to decide if the policies require retraining. 
We point out that as both policy learning and samplingbased verification schemes are done offline prior to deployment of the approximated controllers, this allows us to train and verify, and potentially re-train, with large sample sizes to ensure accuracy of the learned policies $\tilde{U}_{\theta_{p}^{*}}(\cdot)$ and $\tilde{\lambda}_{\theta_{p}^{*}}(\cdot)$.

\section{Online Feasibility and Optimality Certification}

Proposition 1 guarantees that the approximated primal and dual policies $\tilde{U}_{\theta_{p}}(\cdot)$ and $\tilde{\lambda}_{\theta_{d}}$ are feasible and nearoptimal with probability at least $1-\epsilon_{p}$ and $1-\epsilon_{d}$. However, Proposition 1 is not able to certify feasibility and optimality of $\tilde{U}_{\theta_{p}^{*}}(\bar{P})$ and $\tilde{\lambda}_{\theta_{p}^{*}}(\bar{P})$ at a given parameter of $\bar{P}$. To address this issue, we next propose a "hard" deterministic certification scheme, which is carried out online during runtime. We begin with the following observation:

Proposition 2: Given a parameter $P$, assume that $\tilde{U}_{\theta_{p}^{*}}(P)$ satisfies $H(P) \tilde{U}_{\theta_{p}^{*}}(P) \leq h(P)$ and $\tilde{\lambda}_{\theta_{d}^{*}}(P) \geq 0$. Then, the suboptimality of $\tilde{U}_{\theta_{p}^{*}}(P)$ is bounded by

$$
p\left(P ; \tilde{U}_{\theta_{p}^{*}}(P)\right)-d\left(P ; \tilde{\lambda}_{\theta_{d}^{*}}(P)\right),
$$

i.e., $p\left(P ; \tilde{U}_{\theta_{p}^{*}}(P)\right)-J^{*}(P) \leq p\left(P ; \tilde{U}_{\theta_{p}^{*}}(x)\right)-d\left(P ; \tilde{\lambda}_{\theta_{d}^{*}}(P)\right)$.

Proof: By assumption, $\tilde{U}_{\theta_{p}^{*}}(P)$ and $\tilde{\lambda}_{\theta_{d}^{*}}(P)$ are primal and dual feasible, respectively. By weak duality (Section IIIB), $d\left(P ; \tilde{\lambda}_{\theta_{d}^{*}}(P)\right) \leq J^{*}(P)$, which concludes the proof.

Proposition 2 provides a computationally efficient way to estimate the suboptimality of the approximated primal policy $\tilde{U}_{\theta_{p}^{*}}(\cdot)$ without solving the optimization problem.

We use Proposition 2 in our framework as follows: Let $\gamma>0$ be the desired maximum suboptimality level. For a given parameter $P$, if (14) is smaller than $\gamma$, then the primal solution $\tilde{U}_{\theta_{p}^{*}}(P)$ is guaranteed to be at most $\gamma$-suboptimal. In this case, the first element of $\tilde{U}_{\theta_{p}^{*}}(P)$ is applied, and the procedure is repeated at the next time step. If (14) is larger than the predetermined suboptimality level $\gamma$, then a backup controller is employed (see Section IV-D for a discussion).

Algorithm 1 summarizes the proposed Primal-Dual Policy Learning scheme. The following theorem bounds the frequency in which the backup controller will be applied.

Theorem 1: Assume that $\tilde{U}_{\theta_{p}^{*}}\left(P_{t}\right)$ and $\tilde{\lambda}_{\theta_{d}^{*}}\left(P_{t}\right)$ satisfy the conditions (12)-13, and let $\beta, \beta_{p}, \beta_{d}, \epsilon, \epsilon_{p}, \epsilon_{d}, N_{p}$ and $N_{d}$ be chosen as in Algorithm 1 1 Then, with confidence at least $1-\beta$, it holds

$$
\begin{aligned}
& \mathbb{P}\left[H(P) \tilde{U}_{\theta_{p}^{*}}(P) \leq h(P), \tilde{\lambda}_{\theta_{d}^{*}}(P) \geq 0,\right. \\
& \left.p\left(P ; \tilde{U}_{\theta_{p}^{*}}(P)\right)-d\left(P ; \tilde{\lambda}_{\theta_{d}^{*}}(P)\right) \leq \gamma\right] \geq 1-\epsilon .
\end{aligned}
$$

Proof: Application of the union bound [33] and Propositions 1 . 2 yields the desired result.

Theorem 11 ensures that, if the primal and dual policies have been validated according to Proposition 11, then the primal policy $\tilde{U}_{\theta_{p}^{*}}(\cdot)$ will generate inputs that are at most $\gamma$-suboptimal with probability at least $(1-\epsilon)$, which can be checked during run-time using the " $[14) \leq \gamma$ " condition.

\section{Discussion}

Computational Complexity: Since the primal-dual policy learning scheme only requires online the evaluation of

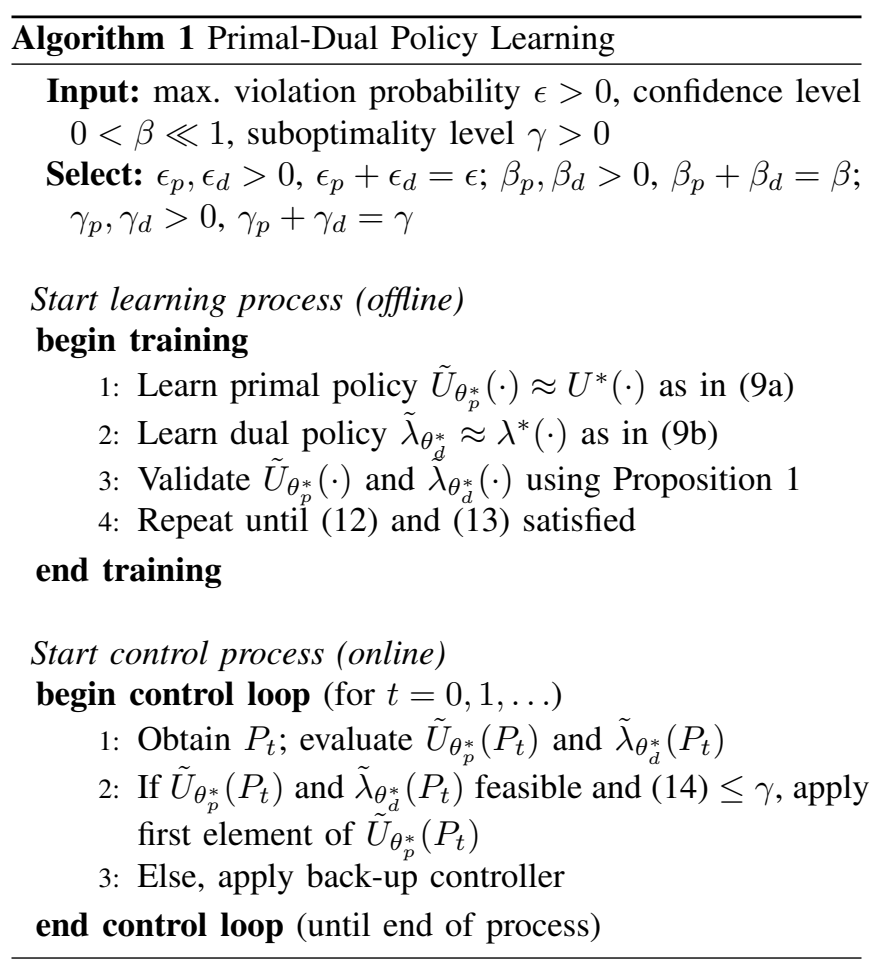

the trained policies $\tilde{U}_{\theta_{p}^{*}}(\cdot)$ and $\tilde{\lambda}_{\theta_{d}^{*}}(\cdot)$, it can typically be executed significantly faster than solving the optimization problem (4). For example, if the policies are approximated through a combination of basis functions, i.e., $\tilde{U}_{\theta}(P)=$ $\sum_{i} \theta_{i} \kappa_{i}(P)$, then the trained policies can be evaluated fully in parallel on embedded platforms such as FPGAs [24].

Backup Controller: The choice of the backup controller, which is used in Algorithm 1 as a fall-back strategy, is highly problem-dependent. Since backup controllers are generally available in practice, we do not see the use of a backup controller as a major limitation of our strategy.

\section{CAse Study: Integrated Chassis Control}

In this section we present a numerical case study in the field of integrated chassis control (ICC) for vehicles. Roughly speaking, the goal in chassis control is to improve a vehicle's dynamics and user comfort by actively controlling its motions. Traditionally, chassis control is carried out by many independent subsystems. More recently, however, there have been attempts to consider the coupling between each individual subsystem, giving rise to so-called integrated chassis control (ICC) [34]. As such, MPC is a natural control strategy, since it allows the incorporation of input and state constraints in a disciplined manner, and is able to handle the multivariate nature of the task.

In the following, we study the problem of calculating the yaw moment, the roll moment and the lateral force in an integrated chassis control problem.

\section{A. Problem Formulation}

We consider a linear parameter-varying system of the form

$x_{t+1}=A\left(v_{t}\right) x_{t}+B\left(v_{t}\right) u_{t}+E\left(v_{t}\right) \delta_{t}, \quad y_{t}=C x_{t}$, 
where $x \in \mathbb{R}^{4}$ is the state, $u \in \mathbb{R}^{3}$ is the input, $y_{t} \in \mathbb{R}^{3}$ is the output, $v_{t} \in \mathbb{R}$ is the vehicle's longitudinal velocity, and $\delta_{t} \in \mathbb{R}$ is the driver's steering input, which we assume can be predicted, see [35] for details on variable nomenclature.

The control objective is to minimize the output tracking error while satisfying input constraints and input rate constraints. Hence, the MPC problem is given by

$$
\begin{array}{cl}
\min _{X_{t}, U_{t}} & \sum_{k=0}^{T-1}\left(y_{k \mid t}-y_{k \mid t}^{\mathrm{ref}}\right)^{\top} Q\left(y_{k \mid t}-y_{k \mid t}^{\mathrm{ref}}\right)+u_{k \mid t}^{\top} R u_{k \mid t} \\
\text { s.t. } & x_{k+1 \mid t}=A\left(v_{t}\right) x_{k \mid t}+B\left(v_{t}\right) u_{k \mid t}+E\left(v_{t}\right) \delta_{k \mid t}, \\
& y_{k \mid t}=C x_{k \mid t},\left|u_{k \mid t}\right| \leq \bar{u},\left|u_{k \mid t}-u_{k-1 \mid t}\right| \leq \overline{\Delta u} \\
& x_{0 \mid t}=x_{t}, u_{-1 \mid t}=u_{t-1}, \quad k=0, \ldots, T-1
\end{array}
$$

where $Q, R \in \mathbb{R}^{3 \times 3}$ are positive definite matrices, $y_{k \mid t}^{\text {ref }} \in$ $\mathbb{R}^{3}$ are given reference signals, $\bar{u}$ defines the input constraints, $\overline{\Delta u}$ defines the rate constraints, and $u_{t-1}$ is the previous input. The parameters in 116 are $P_{t}=$ $\left(x_{t}, v_{t},\left\{y_{k \mid t}^{\text {ref }}, \delta_{k \mid t}\right\}_{k}, u_{-1 \mid t}\right) \in \mathbb{R}^{20}$. The velocity $v_{t}$ enters the dynamics in a nonlinear fashion, and so, the optimal control law $U^{*}\left(P_{t}\right)$ cannot be derived using standard methods from explicit MPC. In the following, we approximate $U^{*}(\cdot)$ using the approach described in Section IV] Throughout, we consider a horizon of $T=3$, which is typical in practice, such that $U^{*}: \mathbb{R}^{20} \rightarrow \mathbb{R}^{9}$.

\section{B. Offline Training and Verification}

In this section, we illustrate our proposed primal-dual policy learning method on (16) using a Deep Neural Network function approximator with Rectified Linear Unit (DNNReLU) activation [36]. We train our primal and dual neural networks using $M=1000$ samples 2 uniformly sampled over a parameter set $\mathcal{P}$. We aim for a maximal admissible suboptimality level of $\gamma=1$, which should hold with probability at least $99 \%$. Following Algorithm 1, we select $\gamma_{p}=\gamma_{d}=\gamma / 2=0.5$ and $\epsilon_{p}=\epsilon_{d}=\epsilon / 2=0.5 \%$. The confidence levels are chosen to be $\beta_{p}=\beta_{d}=10^{-7}$, resulting in a verification sample size of $N_{p}=N_{d}=3216$ (Proposition 11). Using trial and error, we found a DNNReLU of width 15 and depth $L=3$ for $\tilde{U}_{\theta_{p}^{*}}(\cdot)$ which satisfies the conditions in Proposition 1] while a DNN-ReLU of width 5 and depth $L=3$ for $\tilde{\lambda}_{\theta_{d}^{*}}(\cdot)$ is found to satisfy the condition in Proposition 1

Once the policies are trained, to estimate their conservatism, we evaluate the suboptimality levels $\alpha_{p}:=$ $p\left(P ; \tilde{U}_{\theta_{p}^{*}}(P)\right)-J^{*}(P), \alpha_{d}:=J^{*}(P)-d\left(P ; \tilde{\lambda}_{\theta_{d}^{*}}(P)\right)$ and $\alpha:=p\left(P ; \tilde{U}_{\theta_{p}^{*}}(P)\right)-d\left(P ; \tilde{\lambda}_{\theta_{d}^{*}}(P)\right)$ for 100 '000 randomly extracted parameters $P^{(i)}$, and also determine the empirical violation probabilities $\hat{\epsilon}_{p}, \hat{\epsilon}_{d}$ and $\hat{\epsilon}$.

We see from Table I that the approximated primal and dual policies result in control laws that are close to optimal, with an median duality gap of $\alpha=0.00083$, and worst-case duality gap of $\alpha=1.2732$. Furthermore, we see that the empirical probability of the duality gap being larger than

\footnotetext{
${ }^{2}$ The training sample size is a tuning parameter whose choice depends on the specific problem instance.
}

TABLE I

EMPIRICAL SUBOPTIMALITY LEVELS AND VIOLATION PROBABILITIES.

\begin{tabular}{l|lll||ccc}
\hline & $\alpha_{p}$ & $\alpha_{d}$ & $\alpha$ & $\hat{\epsilon}_{p}$ & $\hat{\epsilon}_{d}$ & $\hat{\epsilon}$ \\
\hline mean & 0.0061 & 0.0129 & 0.0190 & & & \\
median & 0.00083 & 0.0053 & 0.0084 & $0.045 \%$ & 0 & $0.005 \%$ \\
maximum & 1.2552 & 0.2608 & 1.2732 & & & \\
\hline
\end{tabular}

the predetermined $\gamma=1$ is $\hat{\epsilon}=0.005 \%$, which is much smaller than the targeted violation probability of $\epsilon=1 \%$. This implies that, for this numerical example, the sample sizes provided by Proposition 1 are conservative; in practice, the trained policies perform better than expected.

\section{Comparison with Online MPC}

In this section, we compare the trained DNN-ReLUs with online MPC in terms of computation time. To solve online MPC, we use Gurobi and Mosek, two state-of-the-art solvers. Table III reports the computation time of our primaldual policy approximation scheme using DNN-ReLU and the online MPC. The timings are taken on an early 2016 MacBook, that runs on a $1.3 \mathrm{GHz}$ Intel Core $\mathrm{m} 7$ processor and is equipped with 8 GB RAM and 512 GB SSD. We use MATLAB generated MEX files to determine the run-time of DNN-ReLU. The optimization problems are formulated in Yalmip [37], and the timings are those reported by the solvers themselves. Notice that, comparing the results with explicit MPC is difficult, since explicit MPC generally only applies for linear time-invariant systems.

TABLE II

COMPUTATION TIMES, ROUNDED TO TWO SIGNIFICANT DIGITS.

\begin{tabular}{l|lll}
\hline time $[\mathrm{ms}]$ & DNN-ReLU & Gurobi & Mosek \\
\hline min. & 0.020 & 1.3 & 2.1 \\
max. & 0.034 & 2.5 & 3.1 \\
mean & 0.023 & 1.5 & 2.4 \\
std. & 0.0023 & 0.25 & 0.23 \\
\hline
\end{tabular}

From Table [I] we see that DNN-ReLU is significantly faster than online MPC, with an average speed-up of over 65x compared to Gurobi, and over 100x compared to Mosek. This is because evaluating a DNN-ReLU just involves simple matrix-vector multiplications and max-operations, but no matrix inversions, as opposed to the case of numerical optimization. Moreover, we observe that the evaluation times obtained using DNN-ReLU are more consistent than those of Gurobi and Mosek, with a standard deviation of $10 \%$ only.

\section{CONCLUSION}

In this paper, we proposed a new method for approximating the explicit MPC control law for linear parameter varying systems. We propose to approximate the MPC controller directly using supervised learning techniques, and invoke two verification schemes to ensure safety and performance of the approximated controller. Since the proposed verification 
scheme only requires the evaluation of trained policies, our algorithm is computationally efficient, and can be implemented even on resource-constrained systems. Indeed, our numerical case study has revealed that the proposed primaldual policy learning framework allows the Integrated Chassis Control problem to be solved up to 100x faster compared to state-of-the-art solvers, while maintaining performance.

\section{ACKNOWLEDGMENT}

This research was partially funded by the Hyundai Center of Excellence at the University of California, Berkeley. This work was also sponsored by the Office of Naval Research. The authors thank Yi-Wen Liao and Dr. Jongsang Suh for helpful discussions on the integrated chassis control problem.

\section{APPENDIX}

\section{Proof of Proposition 1}

Let $H_{j}(P)$ and $h_{j}(P)$ denote the $j$ th row of $H(P)$ and $h(P)$, respectively. For a given $\tilde{U}(\cdot)$, consider the following auxiliary function

$$
\begin{array}{r}
Q(P):=\max \left\{\max _{j}\left\{H_{j}(P) \tilde{U}(P)-h_{j}(P)\right\},\right. \\
\left.p(P ; \tilde{U}(P))-J^{*}(P)-\gamma_{p}\right\},
\end{array}
$$

and define $\hat{Q}_{N}:=\max _{i=1, \ldots, N}\left\{Q\left(P^{(i)}\right)\right\}$, where $\left\{P^{(i)}\right\}_{i}$ are a collection of independent samples drawn according to $\mathbb{P}$. It follows [31, Theorem 3.1] that, if $N \geq \frac{\ln 1 / \beta}{\ln 1 /(1-\epsilon)}$, then

$$
\mathbb{P}^{N}\left[\mathbb{P}\left[Q(P)>\hat{Q}_{N}\right] \leq \epsilon\right] \geq 1-\beta
$$

Proposition 1 now follows from the observation that $\hat{Q}_{N}=0$ is equivalent to 11a).

\section{REFERENCES}

[1] F. Oldewurtel, A. Parisio, C. Jones, D. Gyalistras, M. Gwerder, V. Stauch, B. Lehmann, and M. Morari, "Use of model predictive control and weather forecasts for energy efficient building climate control," Energy and Buildings, vol. 45, pp. 15-27, 2012.

[2] X. Zhang, G. Schildbach, D. Sturzenegger, and M. Morari, "Scenario-based MPC for Energy-Efficient Building Climate Control under Weather and Occupancy Uncertainty," in European Control Conference, 2013, pp. 1029-1034.

[3] Y. Ma, J. Matusko, and F. Borrelli, "Stochastic Model Predictive Control for Building HVAC Systems: Complexity and Conservatism," IEEE Transactions on Control Systems Technology, vol. 23, no. 1, pp. 101116, 2014.

[4] P. Bouffard, A. Aswani, and C. Tomlin, "Learningbased model predictive control on a quadrotor: Onboard implementation and experimental results," in 2012 IEEE International Conference on Robotics and Automation, 2012, pp. 279-284.

[5] A. Liniger, A. Domahidi, and M. Morari, "Optimization-based autonomous racing of 1:43 scale RC cars," Optimal Control Applications and Methods, vol. 36, no. 5, pp. 628-647, 2015.
[6] J. Carrau, A. Liniger, X. Zhang, and J. Lygeros, "Efficient Implementation of Randomized MPC for Miniature Race Cars," in European Control Conference, 2016, pp. 957-962.

[7] U. Rosolia, A. Carvalho, and F. Borrelli, "Autonomous racing using learning model predictive control," in American Control Conference (ACC), 2017, 2017.

[8] M. Bujarbaruah, Z. Ercan, V. Ivanovic, H. E. Tseng, and F. Borrelli, "Torque based lane change assistance with active front steering," in IEEE Intelligent Transportation Systems (ITSC), 2017, pp. 1-6.

[9] X. Zhang, A. Liniger, and F. Borrelli, "OptimizationBased Collision Avoidance," ArXiv preprint arXiv:1711.03449, 2017.

[10] M. Bujarbaruah, X. Zhang, H. Tseng, and F. Borrelli, "Adaptive MPC for Autonomous Lane Keeping," in 14th International Symposium on Advanced Vehicle Control (AVEC), July 2018.

[11] T. Zhang, G. Kahn, S. Levine, and P. Abbeel, "Learning deep control policies for autonomous aerial vehicles with mpc-guided policy search," in IEEE International Conference on Robotics and Automation (ICRA), 2016, pp. 528-535.

[12] J. Mattingley and S. Boyd, "CVXGEN: a code generator for embedded convex optimization," Optimization and Engineering, vol. 13, no. 1, pp. 1-27, 2012.

[13] B. Stellato, G. Banjac, P. Goulart, A. Bemporad, and S. Boyd, "OSQP: An operator splitting solver for quadratic programs," ArXiv e-prints, Nov. 2017. arXiv: 1711.08013 [math.OC].

[14] F. Borrelli, A. Bemporad, and M. Morari, Predictive Control for linear and hybrid systems. Cambridge University Press, 2017.

[15] M. Kvasnica and M. Fikar, "Clipping-based complexity reduction in explicit mpc," IEEE Transactions on Automatic Control, vol. 57, no. 7, pp. 1878-1883, 2012.

[16] T. A. Johansen and A. Grancharova, "Approximate explicit constrained linear model predictive control via orthogonal search tree," IEEE Transactions on Automatic Control, vol. 48, no. 5, pp. 810-815, 2003.

[17] S. Summers, C. N. Jones, J. Lygeros, and M. Morari, "A multiresolution approximation method for fast explicit model predictive control," IEEE Transactions on Automatic Control, vol. 56, no. 11, pp. 2530-2541, 2011.

[18] C. N. Jones and M. Morari, "Polytopic approximation of explicit model predictive controllers," IEEE Transactions on Automatic Control, vol. 55, no. 11, pp. 2542-2553, 2010.

[19] M. Kvasnica and M. Fikar, "Clipping-based complexity reduction in explicit mpc," IEEE Transactions on Automatic Control, vol. 57, no. 7, pp. 1878-1883, 2012. 
[20] M. Kvasnica, J. Hledk, I. Rauov, and M. Fikar, "Complexity reduction of explicit model predictive control via separation," Automatica, vol. 49, no. 6, pp. 1776 $-1781,2013$.

[21] T. Parisini and R. Zoppoli, "A receding-horizon regulator for nonlinear systems and a neural approximation," Automatica, vol. 31, no. 10, pp. $1443-1451$, 1995.

[22] S. Chen, K. Saulnier, N. Atanasov, D. D. Lee, V. Kumar, G. J. Pappas, and M. Morari, "Approximating explicit model predictive control using constrained neural networks," in 2018 Annual American Control Conference (ACC), 2018, pp. 1520-1527.

[23] A. Bemporad, A. Oliveri, T. Poggi, and M. Storace, "Ultra-fast stabilizing model predictive control via canonical piecewise affine approximations," IEEE Transactions on Automatic Control, vol. 56, no. 12, pp. 2883-2897, 2011.

[24] A. Domahidi, M. N. Zeilinger, M. Morari, and C. N. Jones, "Learning a feasible and stabilizing explicit model predictive control law by robust optimization," in 2011 50th IEEE Conference on Decision and Control and European Control Conference, 2011, pp. 513519.

[25] B. Karg and S. Lucia, "Efficient representation and approximation of model predictive control laws via deep learning," ArXiv e-prints, Jun. 2018. arXiv: 1806 . 10644

[26] M. Hertneck, J. Khler, S. Trimpe, and F. Allgwer, "Learning an approximate model predictive controller with guarantees," IEEE Control Systems Letters, vol. 2, no. 3, pp. 543-548, 2018.

[27] P. Goulart, E. Kerrigan, and J. Maciejowski, "Optimization over state feedback policies for robust control with constraints," Automatica, vol. 42, no. 4, pp. 523533, 2006.

[28] A. J. Smola and B. Schölkopf, "A tutorial on support vector regression," Statistics and Computing, vol. 14, no. 3, pp. 199-222, 2004.

[29] M. G. Genton, "Classes of kernels for machine learning: A statistics perspective," J. Mach. Learn. Res., vol. 2, pp. 299-312, Mar. 2002.

[30] S. Boyd and L. Vandenberghe, Convex Optimization. Cambridge University Press, 2004.

[31] R. Tempo, E. Bai, and F. Dabbene, "Probabilistic robustness analysis: Explicit bounds for the minimum number of samples," Systems \& Control Letters, vol. 30, no. 5, pp. $237-242,1997$.

[32] R. Tempo, G. Calafiore, and F. Dabbene, Randomized Algorithms for Analysis and Control of Uncertain Systems. Springer, 2013.

[33] C Bonferroni, "Teoria statistica delle classi e calcolo delle probabilita," Pubblicazioni del R Istituto Superiore di Scienze Economiche e Commericiali di Firenze, vol. 8, pp. 3-62, 1936.

[34] C. Rengaraj and D. Crolla, "Integrated chassis control to improve vehicle handling dynamics performance," in SAE 2011 World Congress \& Exhibition, SAE International, 2011.

[35] S. Takano, M. Nagai, T. Taniguchi, and T. Hatano, "Study on a vehicle dynamics model for improving roll stability," JSAE Review, vol. 24, no. 2, pp. 149 $-156,2003$.

[36] I. Goodfellow, Y. Bengio, and A. Courville, Deep learning. MIT Press, 2016.

[37] J. Löfberg, "YALMIP: A toolbox for modeling and optimization in MATLAB," in IEEE International Symposium on Computer Aided Control Systems Design, 2004, 284-289. 